\begin{tabular}{|c|l|}
\hline Title & Electronic structures of the stage 1 hydrogen- potassium-graphite ternary intercal ation compounds C4KHx \\
\hline Author(s) & Mizuno, Seiji; Nakao, Kenji \\
\hline Citation & $\begin{array}{l}\text { Physical Review B, 41(8), 4938-4947 } \\
\text { https://doi.org/40.1103/PhysRevB.41.4938 }\end{array}$ \\
\hline Issue Date & 1990-03 \\
\hline Doc URL & http://hdl.handle.net/2115/51000 \\
\hline Rights & ○ 1990 The A merican Physical Society \\
\hline Type & article \\
\hline File Information & Phys. Rev. B 41 4938.pdf \\
\hline
\end{tabular}

Instructions for use 


\title{
Electronic structures of the stage-1 hydrogen-potassium-graphite ternary intercalation compounds $\mathrm{C}_{4} \mathrm{KH}_{x}$
}

\author{
Seiji Mizuno and Kenji Nakao \\ Institute of Materials Science, University of Tsukuba, Tsukuba, Ibaraki 305, Japan
}

(Received 2 October 1989)

\begin{abstract}
The electronic structures of the stage-1 hydrogen-potassium-graphite ternary intercalation compounds $\mathrm{C}_{4} \mathrm{KH}_{x}$ are investigated. The band structures of these compounds are determined in a Hohenberg-Kohn-Sham local-density-functional formalism using the self-consistent numericalbasis-set linear combination of atomic-orbitals method. Our band-structure calculation is carried out for $\mathrm{C}_{4} \mathbf{K H}_{x}$ with three assumed in-plane superlattice models of hydrogen structure: (a) $(2 \times 2) R\left(0^{\circ}\right)$ model $(x=0.5)$; (b) $(2 \times \sqrt{3}) R\left(0^{\circ}, 30^{\circ}\right)$ model $(x=0.5)$; (c) $(1 \times \sqrt{3}) R\left(0^{\circ}, 30^{\circ}\right)$ model $(x=1.0)$. In these three cases, we estimate the amount of the charge transfer among the individual atoms and show that the hydrogen atom acts as an acceptor to graphite, while the potassium atom acts as a donor. The charge transfer to the hydrogen atoms is imperfect and therefore the hydrogen $1 s$ state forms a partially occupied metallic band. This hydrogen $1 s$-like band coexists with the graphite $\pi^{*}$-like bands at the Fermi level. These results indicate that $\mathrm{C}_{4} \mathrm{KH}_{x}$ has features like those of two-dimensional metallic hyrodgen.
\end{abstract}

\section{INTRODUCTION}

Recently, much attention has been devoted to the hydrogen-potassium-graphite ternary graphite intercalation compounds ( $\mathrm{KH}_{x}$ GIC's). ${ }^{1-13}$ The stage-1 and -2 compounds have the chemical formulas $\mathrm{C}_{4} \mathrm{KH}_{x}$ and $\mathrm{C}_{8} \mathrm{KH}_{x}$, respectively.

The structure and electronic properties of these $\mathrm{KH}_{x}$ GIC's have been investigated by many experiments. ${ }^{1-11}$ However, there are some controversial discussions about them. As for the electronic structure, two different models are proposed from experiments. Two schematic pictures of the density of states are shown in Figs. 1(a) and 1(b). In the figures, the potassium $4 s$ band, hydrogen $1 s$ band, and graphite upper and lower $\pi$ bands are shown. In the model (a), the potassium $4 s$ band lies above the Fermi level and therefore the charge transfer from this potassium band is perfect. On the other hand, the hydrogen $1 s$ band lies below the Fermi level and the charge transfer to the hydrogen band is perfect. This result indicates that the hydrogen atom in these compounds has a localized electronic state. This model (a) is proposed from low-temperature specific heat, ${ }^{1}$ Shubnikov-de hass effect, ${ }^{2}$ optical reflectivity, ${ }^{3}$ and so on. In the model (b), on the other hand, the hydrogen $1 \mathrm{~s}$ state intersects the Fermi level and forms a metallic band. This model (b) is proposed from recent experimental results of proton NMR, ${ }^{4,5}$ electrical conductivity, ${ }^{6}$ thermoelectric power, ${ }^{6}$ and ESR, ${ }^{1} \mathrm{H}$ NMR, and ${ }^{13} \mathrm{C} \mathrm{NMR}{ }^{7}$ That is, the central problem is whether the hydrogen $1 \mathrm{~s}$ state lies below the Fermi level or forms a metallic band intersecting the Fermi level.

In a previous paper, ${ }^{12,13}$ we calculate the band structure of the stage- 2 compound $\mathrm{C}_{8} \mathrm{KH}_{x}$ and show that the hydrogen $1 s$ state forms a metallic band. That is, this result supports the electronic structure model (b) (see Fig. 1).
In the present paper, we present the calculated band structure of the stage- 1 compound $\mathrm{C}_{4} \mathrm{KH}_{x}$. Though it is known that this compound has a structure where potassium-hydrogen-potassium sandwiched layers are formed in the intercalant, the in-plane structure of a hydrogen layer has not been established. Therefore, in this study we use three simple models: $(2 \times 2) R\left(0^{\circ}\right)$; $(2 \times \sqrt{3}) R\left(0^{\circ}, 30^{\circ}\right) ;(1 \times \sqrt{3}) R\left(0^{\circ}, 30^{\circ}\right)$ in-plane superlattice model (see Fig. 2). Our band-structure calculation is carried out for $\mathrm{C}_{4} \mathbf{K H}_{x}$ with these three model structures. Our results show that the hydrogen $1 s$ state forms a metallic band in $\mathrm{C}_{4} \mathrm{KH}_{x}$, as in the stage- $2 \mathrm{C}_{8} \mathrm{KH}_{x}$.

Further, we discuss the feature of the band structure near the Fermi level, using simple tight-binding models. As a result, it is found that the charge imbalance between the nonequivalent carbon atoms in a graphite layer is important for understanding the separation of the $\pi^{*}$ bands near the Fermi level around the $\Gamma$ point of the Brillouin zone in Fig. 3.

The outline of this paper is as follows. In Sec. II, the crystal structures chosen for this study are discussed. In Sec. III, we present the band structures of $\mathrm{C}_{4} \mathrm{KH}_{x}$ obtained by the self-consistent calculation, and compare these band structures with the rigid band derived from the band structure of graphite. Further, we discuss the feature of the band structure near the Fermi level, using simple tight-binding models. In Sec. IV, a summary is given.

\section{CRYSTAL STRUCTURE}

$\mathrm{C}_{4} \mathrm{KH}_{x}$ has a stage-1 structure where $\mathrm{K}-\mathrm{H}-\mathrm{K}$ sandwiched layers are formed in the intercalants. That is, the stacking order along the $c$ axis is $-\mathbf{C}-\mathbf{K}-\mathbf{H}-\mathbf{K}-\mathbf{C}-$. The $c$ axis repeat distance is reported to be $8.53 \AA{ }^{8}$ The neutron scattering experiments by Kamitakahara et al. ${ }^{9}$ indicate a stacking sequence 
$A \phi B \chi A \phi$, where $A, B$ denote the graphite layers and $\phi, \chi$ denote the $\mathrm{K}-\mathrm{H}-\mathrm{K}$ trilayers. For simplicity, in this study we use the simpler stacking sequence $A \phi A \phi A \phi$. This difference is not important because the interaction between layers is expected to be very weak. [In fact, our calculated results show that the energy band near the Fermi level has very small $c$-axis dispersion (Fig. 4).]

As for the in-plane atomic arrangement, the structure
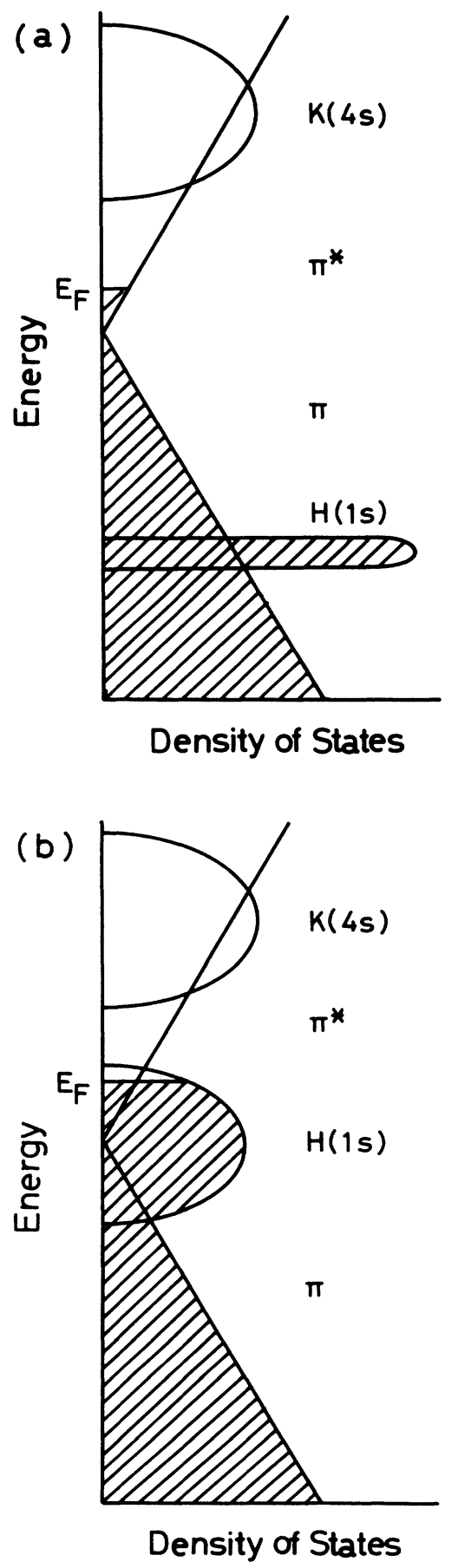

FIG. 1. Two models of the density of states for the $\mathrm{KH}_{x}$ GIC's. of a carbon layer is a hexagonal net as in graphite. In a potassium layer, potassium atoms form a $(2 \times 2) R\left(0^{\circ}\right) \mathrm{su}-$ perlattice. However, the structure of a hydrogen layer is not established to date. Some possible models are proposed. ${ }^{1,10,11}$ In this study, we use three simple models: (a) $(2 \times 2) R\left(0^{\circ}\right) ; \quad$ (b) $(2 \times \sqrt{3}) R\left(0^{\circ}, 30^{\circ}\right)$; (c) $(1 \times \sqrt{3}) R\left(0^{\circ}, 30^{\circ}\right)$ in-plane superlattice model. Our band-structure calculation is carried out for $\mathrm{C}_{4} \mathrm{KH}_{x}$ with these assumed structures.

\section{A. $(2 \times 2) R\left(0^{\circ}\right)$ in-plane superlattice model}

Figure 2(a) shows the $(2 \times 2) R\left(0^{\circ}\right)$ in-plane superlattice model of hydrogen structure. This model corresponds to a hydrogen content $x=0.5$. The Bravais lattice of this model structure is base-centered orthorhombic and its space group is $D_{2}^{6}(C 222)$. The primitive translation vectors are

$$
\begin{aligned}
& \mathbf{a}=(a / 2,-\sqrt{3} a / 2,0), \\
& \mathbf{b}=(a / 2, \sqrt{3} a / 2,0), \\
& \mathbf{c}=(0,0, c),
\end{aligned}
$$

where $a=4.92 \AA$ and $c=8.53 \AA$. The $x$ and $y$ axes are shown in Fig. 2(a) and the $z$ axis is perpendicular to the layer plane. A unit cell is denoted by dashed lines in this figure. The unit cell contains 11 atoms: 8 carbon atoms, 2 potassium atoms, and 1 hydrogen atom. The carbon atoms are classified into three environmentally nonequivalent types by symmetry, and the two potassium atoms are equivalent. The three carbon atoms are denoted by numbers 1, 2, and 3 in Fig. 2(a). The carbon 1 has one nearest-neighbor potassium atom in the adjacent potassium layer. On the other hand, the carbon 2 and 3 have two nearest-neighbor potassium atoms in the adjacent potassium layers. In the present calculation, we take account of the charge transfer among these nonequivalent atoms completely.

The reciprocal-lattice vectors are

$$
\begin{aligned}
& \mathbf{a}^{*}=(2 \pi / a,-2 \pi / \sqrt{3} a, 0), \\
& \mathbf{b}^{*}=(2 \pi / a, 2 \pi / \sqrt{3} a, 0), \\
& \mathbf{c}^{*}=(0,0,2 \pi / c) .
\end{aligned}
$$

The Brillouin zone (BZ) defined by these reciprocallattice vectors is a hexagonal prism, as in graphite. This is shown in Fig. 3(a).

\section{B. $(2 \times \sqrt{3}) R\left(0^{\circ}, 30^{\circ}\right)$ in-plane superlattice model}

Figure 2(b) shows the $(2 \times \sqrt{3}) R\left(0^{\circ}, 30^{\circ}\right)$ in-plane superlattice model of hydrogen structure. This model (b) corresponds to a hydrogen content $x=0.5$, as the model (a). The Bravais lattice of this model structure is different from that of the model (a). It is primitive orthorhombic and its space group is $D_{2}^{1}(p 222)$. The primitive translation vectors are

$$
\begin{aligned}
& \mathbf{a}=(a, 0,0), \\
& \mathbf{b}=(0, \sqrt{3} a, 0), \\
& \mathbf{c}=(0,0, c) .
\end{aligned}
$$




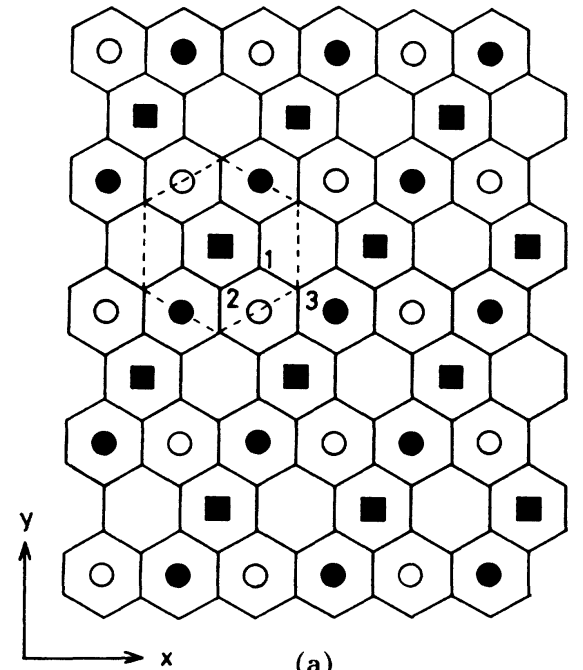

(a)

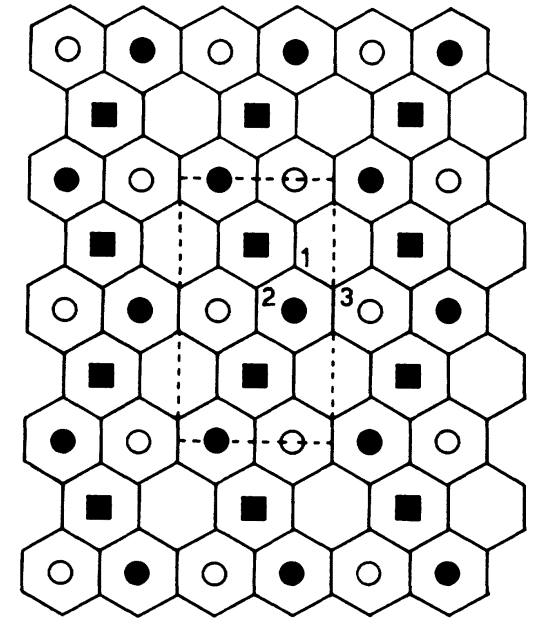

(b)

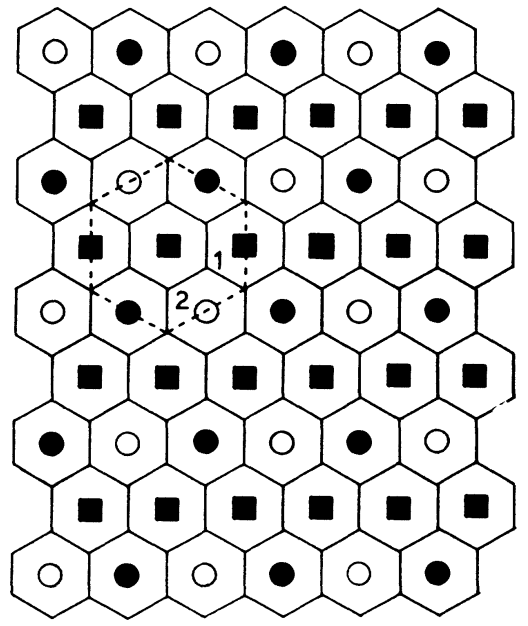

(c)

FIG. 2. Top view of the structural models: (a) $(2 \times 2) R\left(0^{\circ}\right)$; (b) $(2 \times \sqrt{3}) R\left(0^{\circ}, 30^{\circ}\right)$; (c) $(1 \times \sqrt{3}) R\left(0^{\circ}, 30^{\circ}\right)$ in-plane superlattice model of hydrogen structure. The hexagonal net represents the carbon layer. Hydrogen atoms are denoted by squares $(\boldsymbol{\square})$. Potassium atoms are denoted by closed circles $(O)$ and open circles $(O)$. The stacking order along the $c$ axis is $-C-K(O)-\mathbf{H}(\square)-\mathbf{K}(O)-C-$. The environmentally nonequivalent carbon atoms are denoted by numbers. Models (a), (b), and (c) correspond to hydrogen contents $x=0.5,0.5$, and 1.0 , respectively.

The unit cell contains 22 atoms: 16 carbon atoms, 4 equivalent potassium atoms, and 2 equivalent hydrogen atoms. The carbon atoms are classified into three environmentally nonequivalent types. These three carbon atoms are denoted by numbers 1, 2, and 3 in Fig. 2(b). The carbon 1 has one nearest-neighbor potassium atom in the adjacent potassium layer. On the other hand, the carbon 2 and 3 have two in the adjacent potassium layers. The reciprocal-lattice vectors are

$$
\begin{aligned}
& \mathbf{a}^{*}=(2 \pi / a, 0,0), \\
& \mathbf{b}^{*}=(0,2 \pi / \sqrt{3} a, 0), \\
& \mathbf{c}^{*}=(0,0,2 \pi / c) .
\end{aligned}
$$

The BZ is a rectangular parallelepiped. This is shown in Fig. 3(b).

\section{C. $(1 \times \sqrt{3}) R\left(0^{\circ}, 30^{\circ}\right)$ in-plane superlattice model}

Figure 2(c) shows the $(1 \times \sqrt{3}) R\left(0^{\circ}, 30^{\circ}\right)$ in-plane superlattice model of hydrogen structure. This model (c) corresponds to a hydrogen content $x=1.0$. The Bravais lattice of this model structure (c) is base-centered orthorhombic and its space group is $D_{2 h}^{21}(\mathrm{cmma})$. The primitive translation vectors and the corresponding reciprocal-lattice vectors are the same as the model (a). The $\mathrm{BZ}$ is a hexagonal prism, as in the model (a) [Fig. 3(a)].

The unit cell contains 12 atoms: 8 carbon atoms, 2 equivalent potassium atoms, and 2 equivalent hydrogen atoms. The carbon atoms are classified into two nonequivalent types. These two carbon atoms are denoted by numbers 1 and 2 in Fig. 2(c). The carbon 1 has one nearest-neighbor potassium atom in the adjacent potassi- um layer. On the other hand, the carbon 2 has two in the adjacent potassium layers.

\section{RESULTS AND DISCUSSION}

In this section, we first present the band structure of $\mathrm{C}_{4} \mathrm{KH}_{x}$ obtained by the self-consistent calculation, and

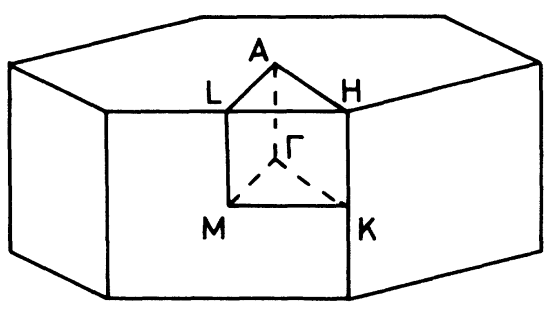

(a)

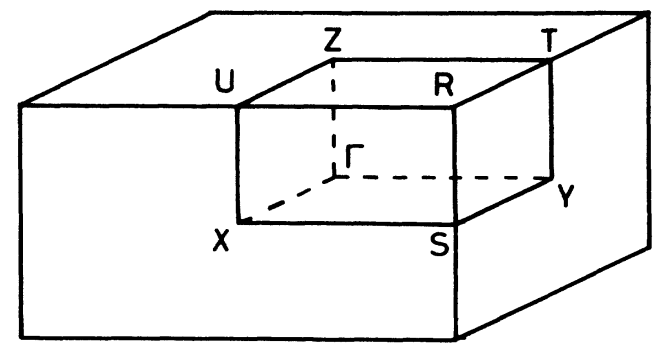

(b)

FIG. 3. The Brillouin zone of $\mathrm{C}_{4} \mathrm{KH}_{x}$. (a) $(2 \times 2) R\left(0^{\circ}\right)$ model and $(1 \times \sqrt{3}) R\left(0^{\circ}, 30^{\circ}\right)$ model. (b) $(2 \times \sqrt{3}) R\left(0^{\circ}, 30^{\circ}\right)$ model. 
discuss main features of the band structure. Next, we reproduce the main features of the band structure, using simple tight-binding models. Further, we discuss the difference between the feature of $\pi$ bands in $\mathrm{C}_{4} \mathrm{KH}_{x}$ and that in the binary GIC $\mathrm{C}_{8} \mathrm{~K}$ and point out the importance of the charge imbalance between the nonequivalent carbon atoms in a graphite layer in these compounds.
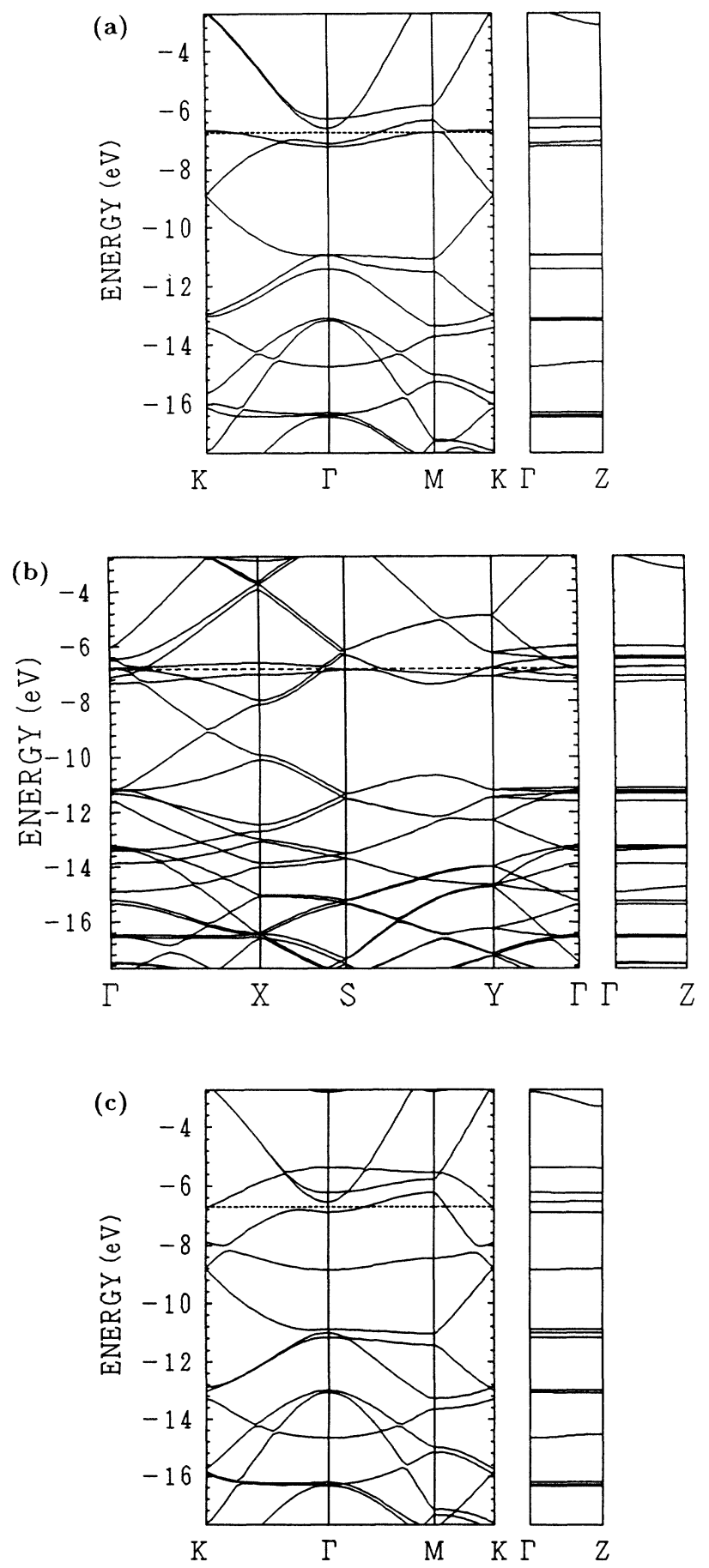

FIG. 4. Self-consistent band structures along several highsymmetry directions in the Brillouin zone: (a) $(2 \times 2) R\left(0^{\circ}\right)$ model; (b) $(2 \times \sqrt{3}) R\left(0^{\circ}, 30^{\circ}\right)$ model; (c) $(1 \times \sqrt{3}) R\left(0^{\circ}, 30^{\circ}\right)$ model. Here, the dashed line represents the Fermi energy.

\section{A. Band-structure calculation}

The numerical method used in the present work is the self-consistent numerical-basis-set linear combination of atomic-orbitals (LCAO) method within the HohenbergKohn-Sham local-density-functional formalism. ${ }^{14,15}$ This method was developed by Zunger and Freeman ${ }^{16}$ and later applied to the band-structure calculation of the binary GIC $\mathrm{C}_{8} \mathrm{~K}$ and the ternary GIC $\mathrm{C}_{8} \mathrm{KH}_{x}$ in a previous paper. ${ }^{12,13,17,18}$ The Hedin-Lundqvist parametrization $^{19}$ for the exchange-correlation potential was used. As a numerical basis set, in this study we take the $1 s, 2 s$, and $2 p$ orbitals of carbon, and $1 s, 2 s, 2 p, 3 s, 3 p$, and $4 s$ orbitals of potassium and $1 s$ orbital of hydrogen. The details of this method are presented in a previous paper. ${ }^{16,18}$ Our band-structure calculation is carried out for $\mathrm{C}_{4} \mathrm{KH}_{x}$ with three assumed structure models (a), (b), and (c).

\section{1. $(2 \times 2) R\left(0^{\circ}\right)$ model $(x=0.5)$}

The calculated band structure of $\mathrm{C}_{4} \mathrm{KH}_{0.5}$ with the structure model (a) is shown in Fig. 4(a). [The model (a) is the $(2 \times 2) R\left(0^{\circ}\right)$ triangular in-plane superlattice model of the hydrogen structure.] The overall band structure is

TABLE I. Occupation numbers.

\begin{tabular}{|c|c|c|}
\hline Atom & Orbitals & $\begin{array}{c}\text { Occupation } \\
\text { number }\end{array}$ \\
\hline \multicolumn{3}{|c|}{ (a) $\mathrm{C}_{4} \mathrm{KH}_{0 \leq}\left[(2 \times 2) R\left(0^{\circ}\right)\right.$ model $]$} \\
\hline \multirow[t]{2}{*}{ Carbon 1} & $2 s$ & 1.20 \\
\hline & $2 p$ & 2.84 \\
\hline \multirow[t]{2}{*}{ Carbon 2} & $2 s$ & 1.24 \\
\hline & $2 p$ & 3.05 \\
\hline \multirow[t]{2}{*}{ Carbon 3} & $2 s$ & 0.86 \\
\hline & $2 p$ & 3.09 \\
\hline Potassium & $4 s$ & 0.54 \\
\hline Hydrogen & $1 s$ & 1.29 \\
\hline \multicolumn{3}{|c|}{ (b) $\mathrm{C}_{4} \mathrm{KH}_{0.5}\left[(2 \times \sqrt{3}) R\left(0^{\circ}, 30^{\circ}\right)\right.$ model $]$} \\
\hline \multirow[t]{2}{*}{ Carbon 1} & $2 s$ & 1.17 \\
\hline & $2 p$ & 2.84 \\
\hline \multirow[t]{2}{*}{ Carbon 2} & $2 s$ & 1.14 \\
\hline & $2 p$ & 3.02 \\
\hline \multirow[t]{2}{*}{ Carbon 3} & $2 s$ & 1.05 \\
\hline & $2 p$ & 3.06 \\
\hline Potassium & $4 s$ & 0.59 \\
\hline \multirow[t]{2}{*}{ Hydrogen } & $1 s$ & 1.26 \\
\hline & (c) $\mathrm{C}_{4} \mathrm{KH}_{10}[(1 \times \sqrt{3}$ & del] \\
\hline \multirow[t]{2}{*}{ Carbon 1} & $2 s$ & 1.22 \\
\hline & $2 p$ & 2.81 \\
\hline \multirow[t]{2}{*}{ Carbon 2} & $2 s$ & 1.04 \\
\hline & $2 p$ & 3.09 \\
\hline Potassium & $4 s$ & 0.53 \\
\hline Hydrogen & $1 s$ & 1.13 \\
\hline
\end{tabular}


similar to that obtained by superposing the hydrogen and potassium bands on graphite bands folded into the smaller $\mathrm{BZ}$ of $\mathrm{C}_{4} \mathrm{KH}_{0.5}$ (the band folding is described in detail in Sec. III B 1). Thus, many of the $\mathrm{C}_{4} \mathbf{K H}_{0.5}$ bands can be identified with the $\pi, \pi^{*}$, and $\sigma$ bands of two-dimensional (2D) graphite. However, the $\pi^{*}$ band near the Fermi level split off around the $\Gamma$ point and one of them goes down below the Fermi level. This separation cannot be understood in terms of the band folding. We will explain this energy separation in Sec. III A 2 using a simple tightbinding model.

The most remarkable feature in this band structure is the existence of two kinds of partially occupied conduction bands. These are directly shown to be a hydrogen $1 s$-like band and a graphite $\pi^{*}$-like band, from the amplitude of the wave function. That is, the hydrogen $1 s$-like band coexists with the graphite $\pi^{*}$-like bands at the Fermi level in $\mathrm{C}_{4} \mathrm{KH}_{0.5}$ with the triangular-lattice model.

The $\mathrm{H} 1 s$-like band has very small $c$-axis dispersion because of a large separation $(8.53 \AA)$ between the nearest hydrogen layers. The in-plane bandwidth of this twodimensional band is about $0.6 \mathrm{eV}$. Thus, the hydrogen in $\mathrm{C}_{4} \mathrm{KH}_{0.5}$ has a weakly metallic nature. Consequently, the 2D hydrogen $1 s$-like Fermi surface around the center of the $\mathrm{BZ}$ coexists with the $2 \mathrm{D}$ graphite $\pi^{*}$-like Fermi surface.

In the present calculation, the atomic occupation numbers are treated essentially as iteration parameters in the self-consistent procedure. The obtained final occupation numbers are shown in Table $I(a)$. The total population of each atom in the crystal is given in Table II(a). We define the amount of the charge redistribution of atoms as the difference between the above self-consistent population and the population of isolated (neutral) atoms. ${ }^{17,18}$ These

TABLE II. Total population and amount of charge redistribution.

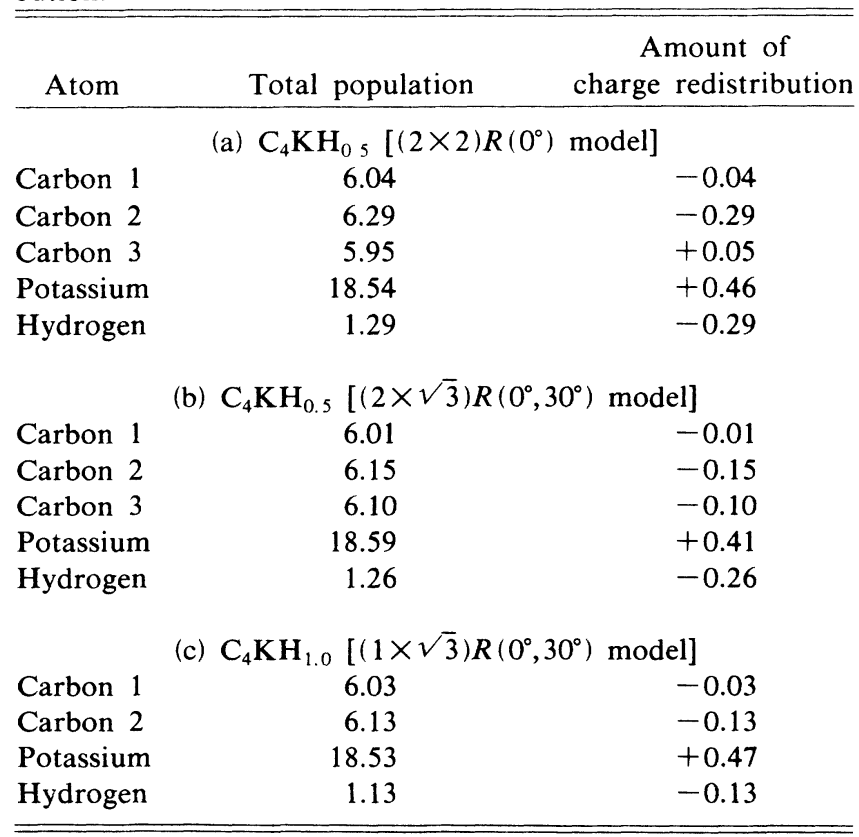

values are also shown in Table II(a). Here, a plus sign represents the charge transfer from the atom and a minus sign represents the transfer to the atom.

These results indicate that the hydrogen atom acts as an acceptor, while the potassium atom acts as a donor. Further, the charge redistribution between the environmentally nonequivalent carbon atoms is produced.

Though the K $4 s$-like bands lie above the Fermi level, the amount of the charge redistribution of potassium atom is not unity. The reason for this is that the $\mathrm{K} 4 \mathrm{~s}$ components are mixed slightly into the wave functions of
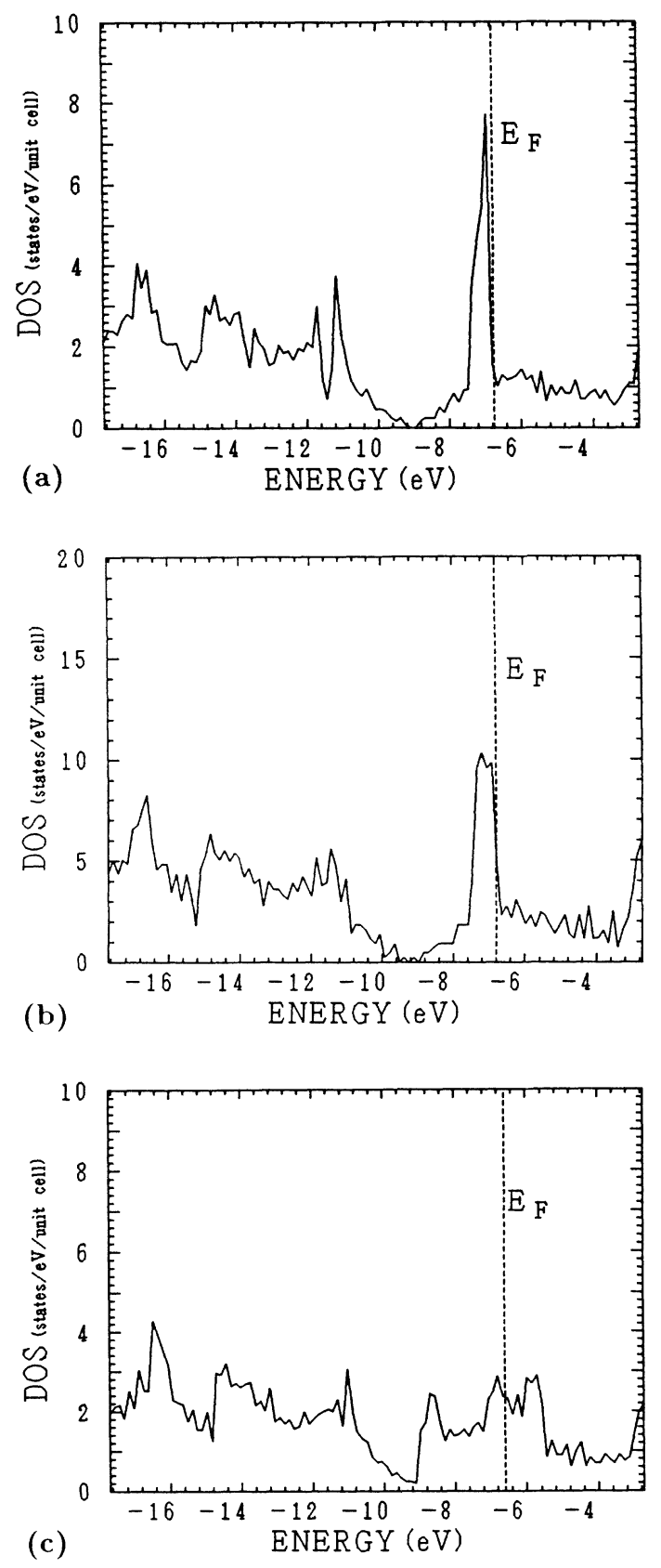

FIG. 5. Density of states of $\mathrm{C}_{4} \mathrm{KH}_{x}$ : (a) $(2 \times 2) R\left(0^{\circ}\right)$ model; (b) $(2 \times \sqrt{3}) R\left(0^{\circ}, 30^{\circ}\right)$ model; (c) $(1 \times \sqrt{3}) R\left(0^{\circ}, 30^{\circ}\right)$ model. 
many graphite-like and hydrogen-like states below the Fermi level.

Figure 5(a) shows the calculated density of states for $\mathrm{C}_{4} \mathrm{KH}_{0.5}$ with the hexagonal-lattice model. The large peak near $0.2 \mathrm{eV}$ below the Fermi level corresponds to the $\mathrm{H} 1 s$ state. This large value is a result of the small dispersion of the $\mathrm{H} 1 s$ band. The density of states at the Fermi level is about 1.3 states/eV per unit cell. The structures near $-11 \mathrm{eV}$ and below $-14 \mathrm{eV}$ correspond to the graphite $\pi$ and $\sigma$ states, respectively.

The present result is very similar to the result of the stage-2 compound $\mathrm{C}_{8} \mathrm{KH}_{0.5}$ with the $(2 \times 2) R\left(0^{\circ}\right)$ hexagonal-lattice model in a previous work. ${ }^{12}$ The only difference is the number of folded graphite bands. In $\mathrm{C}_{8} \mathrm{KH}_{0.5}$, a unit cell contains two graphite layers and therefore there are two folded graphite bands. Due to the interaction between the graphite layers, two folded bands split off.

\section{2. $(2 \times \sqrt{3}) R\left(0^{\circ}, 30^{\circ}\right)$ model $(x=0.5)$}

The calculated band structure of $\mathrm{C}_{4} \mathrm{KH}_{0.5}$ with the structure model (b) is shown in Fig. 4(b). [The model (b) is the $(2 \times \sqrt{3}) R\left(0^{\circ}, 30^{\circ}\right)$ rectangular in-plane superlattice model of the hydrogen structure.] The overall band structure is similar to that obtained by superposing the hydrogen and potassium bands on graphite bands folded into the smaller $\mathrm{BZ}$ of $\mathrm{C}_{4} \mathrm{KH}_{0.5}$, as in the model (a).

This band structure seems to be quite different from that of the model (a). This is due to different shape and size of the BZ. In fact, this band structure can be well identified with the $\pi, \pi^{*}$, and $\sigma$ bands as in the model (a), using the rigid-band model. The folding of the $\pi$ and $\pi^{*}$ bands in this case is described in detail in Sec. III B 1.

In this model structure, the unit cell contains two hydrogen atoms. Therefore, there are bonding and antibonding $\mathbf{H ~} 1 s$-like bands. The bonding $\mathbf{H} 1 s$-like band lies below the Fermi level and the antibonding $\mathrm{H} 1 s$-like band intersects the Fermi level (these two bands are degenerate on the $S$ - $Y$ line). Therefore, it is found that the $\mathrm{H} 1 s$-like band coexists with the $\pi^{*}$-like bands at the Fermi level in the model (b), as in the model (a).

The bonding and antibonding $\mathrm{H} 1 s$-like bands have very small $c$-axis dispersion because of the large separation between the nearest hydrogen layers. The in-plane bandwidth of the bonding and antibonding $\mathrm{H} 1 \mathrm{~s}$ bands are about 0.5 and $0.2 \mathrm{eV}$, respectively. The sum of these two values is close to the bandwidth of the $\mathrm{H} 1 s$ band in $\mathrm{C}_{4} \mathrm{KH}_{0.5}$ with the model (a). Thus, the hydrogen in this crystal also has a weakly metallic nature.

Consequently, it is found that the band structures of $\mathrm{C}_{4} \mathrm{KH}_{0.5}$ with the $(2 \times \sqrt{3}) R\left(0^{\circ}, 30^{\circ}\right)$ rectangular-lattice model has the essentially same feature as that with the $(2 \times 2) R\left(0^{\circ}\right)$ hexagonal-lattice model, though it seems to be different at sight.

The obtained final occupation numbers are shown in Table I(b). The total population of each atom in $\mathrm{C}_{4} \mathrm{KH}_{0.5}$ is given in Table II(b). The amount of the charge redistribution is also shown in Table II(b). These values indicate that the hydrogen atom acts as an acceptor and the potassium atom acts as a donor. The charge redistribution between the nonequivalent carbon atoms is also produced.

Figure 5(b) shows the calculated density of states for $\mathrm{C}_{4} \mathrm{KH}_{0.5}$. The large peak near $0.4 \mathrm{eV}$ below the Fermi level corresponds to the $\mathrm{H} 1 s$ state. This large value is a result of the small dispersion of the $\mathrm{H} 1 s$ band. The density of states at the Fermi level is about 5.0 states/eV per unit cell. The structures near $-11 \mathrm{eV}$ and below $-14 \mathrm{eV}$ correspond to the graphite $\pi$ and $\sigma$ states, respectively.

The structure of the density of states is very similar to that of the model (a) [Fig. 5(a)]. The density of states per unit cell has twice the value of that of model (a). This is due to the fact that the unit cell of the model (b) contains twice the number of atoms of the model (a).

The models (a) and (b) correspond a hydrogen content $x=0.5$. Therefore, it is found that the electronic state of $\mathrm{C}_{4} \mathrm{KH}_{0.5}$ is almost independent on the hydrogen structure.

\section{3. $(1 \times \sqrt{3}) R\left(0^{\circ}, 30^{\circ}\right)$ model $(x=1.0)$}

The calculated band structure of $\mathrm{C}_{4} \mathrm{KH}_{1.0}$ with the structure model (c) is shown in Fig. 4(c). [The model (c) is $(1 \times \sqrt{3}) R\left(0^{\circ}, 30^{\circ}\right)$ in-plane superlattice model of the hydrogen structure.]

In $\mathrm{C}_{4} \mathrm{KH}_{1.0}$, the unit cell contains two hydrogen atoms. Therefore, there are bonding and antibonding $\mathrm{H} 1 s$-like bands. The overall band structure of $\mathrm{C}_{4} \mathrm{KH}_{1.0}$ is also similar to that obtained by superposing the bonding and antibonding hydrogen bands on the graphite bands folded into the $\mathrm{BZ}$ of $\mathrm{C}_{4} \mathrm{KH}_{1.0}$. The antibonding $\mathrm{H} 1 s$-like band intersects the Fermi level near the $K$ point. The bonding $\mathrm{H} 1 s$-like band lies about $2.0 \mathrm{eV}$ below the Fermi level. Therefore, it is found that the $\mathrm{H} 1 s$-like band coexists with the $\pi^{*}$-like bands at the Fermi level in $\mathrm{C}_{4} \mathrm{KH}_{1.0}$, as in $\mathrm{C}_{4} \mathrm{KH}_{0.5}$. The $\pi^{*}$ band near the Fermi level split off around the $\Gamma$ point and one of them goes down below the Fermi level, as in the model (a). This separation cannot be understood in terms of the band folding. We will explain this energy separation in Sec. III B 2 using a simple tight-binding model.

The bonding and antibonding $\mathrm{H} 1 s$-like bands have very small $k_{z}$ dispersion because of a large separation between the nearest hydrogen layers. The in-plane bandwidth of the bonding and antibonding $\mathrm{H} 1 \mathrm{~s}$ bands are about 1.0 and $1.3 \mathrm{eV}$, respectively. These are larger than the bandwidth of the $\mathrm{H} 1 s$ band in $\mathrm{C}_{4} \mathrm{KH}_{0.5}$. This is due to the higher density of hydrogen. In our model structure of $\mathrm{C}_{4} \mathrm{KH}_{1.0}$, the hydrogen atoms form linear chains along the $x$ axis. Therefore, the dispersion of the antibonding $\mathrm{H} 1 s$ band has a one-dimensional (1D) feature. Thus, the hydrogen in $\mathrm{C}_{4} \mathrm{KH}_{1.0}$ has a $1 \mathrm{D}$ metallic nature. Consequently, the 1D H $1 s$-like Fermi surfaces in the corners of the BZ coexists with the $2 \mathrm{D}$ graphite $\pi^{*}$-like Fermi surfaces.

The obtained final occupation numbers are shown in Table I(c). The total population of each atom in $\mathrm{C}_{4} \mathrm{KH}_{1.0}$ is given in Table II(c). The amount of the charge redistribution is also shown in Table II(c). These values indicate that the hydrogen atom acts as an acceptor and the potassium atom acts as a donor. The charge redistribution 
between the nonequivalent carbon atoms is also produced.

Figure 5(c) shows the calculated density of states for $\mathrm{C}_{4} \mathrm{KH}_{1.0}$. In $\mathrm{C}_{4} \mathrm{KH}_{1.0}$, there are two structures corresponding to $\mathrm{H} 1 s$ states; the structures near -8.5 and $-5.8 \mathrm{eV}$ correspond to the bonding and antibonding states, respectively. The density of states at the Fermi level is about 2.4 states/eV per unit cell.

The present result is very similar to the result of the stage- 2 compound $\mathrm{C}_{8} \mathrm{KH}_{1.0}$ with the $(1 \times \sqrt{3}) R\left(0^{\circ}, 30^{\circ}\right)$ linear chains lattice model in a previous work. ${ }^{13}$ The only difference is the number of the folded graphite bands. In $\mathrm{C}_{8} \mathrm{KH}_{1.0}$, a unit cell contains two graphite layers. Therefore, there are two folded graphite bands. Further, these bands split off due to the interaction between the graphite layers.

Our results show that the electronic structure of the stage- 1 compound $\mathrm{C}_{4} \mathrm{KH}_{x}$ is very similar to that of the stage- 2 compound $\mathrm{C}_{8} \mathrm{KH}_{x}$. That is, the hydrogen $1 s$ state forms a metallic band intersecting the Fermi level; this result supports the electronic structure model (b) [Fig. 1(b)]. Now, we will discuss experimental results in terms of our results. Enoki et al. ${ }^{6}$ measured electrical conductivity and thermoelectric power of the stage- 1 and stage- 2 compounds. They showed that potassium acts as a donor to graphite, while hydrogen acts as an acceptor, and the hydrogen has a weakly metallic nature in these compounds. This result is consistent with our results. Other experiments [proton NMR, ${ }^{4,5}$ and ESR, ${ }^{1} \mathrm{H} \mathrm{NMR}$, and ${ }^{13} \mathrm{C}$ NMR (Ref. 7)] supporting the electronic structure model (b) [Fig. 1(b)] are carried out only for the stage-2 compound.

Very recently, Miyajima et al. ${ }^{20}$ carried out a proton NMR study on the stage- 1 compound $\mathrm{C}_{4} \mathrm{KH}_{x}$. They observed metallic nature of hydrogen through the Korringa relation, $T_{1} T=$ const, for the spin-lattice relaxation $T_{1}$. This is also consistent with our results.

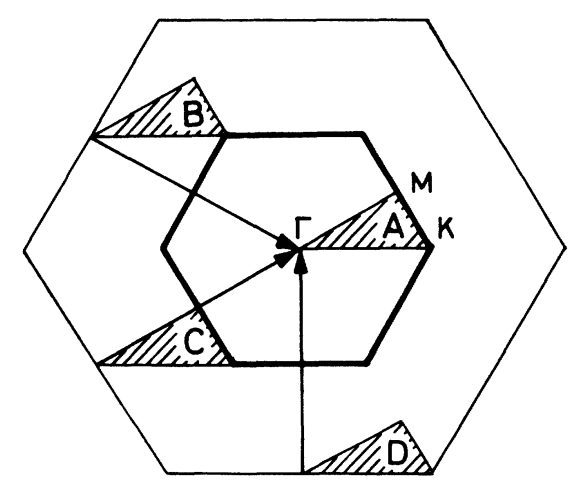

FIG. 6. Two-dimensional $\mathrm{BZ}$ of $\mathrm{C}_{4} \mathrm{KH}_{x}$ with the $(2 \times 2) R\left(0^{\circ}\right)$ or $(1 \times \sqrt{3}) R\left(0^{\circ}, 30^{\circ}\right)$ model (thick solid line) and $\mathrm{BZ}$ of $2 \mathrm{D}$ graphite (thin solid line). The four hatched regions are indicated as $A, B, C$, and $D$. Region $A$ is in the first $\mathrm{BZ}$ of $\mathrm{C}_{4} \mathrm{KH}_{x}$ and regions $B, C$, and $D$ are out of this BZ. Regions $B, C$, and $D$ are all transferred to region $A$ by proper reciprocal-lattice vectors of $\mathrm{C}_{4} \mathrm{KH}_{x}$.

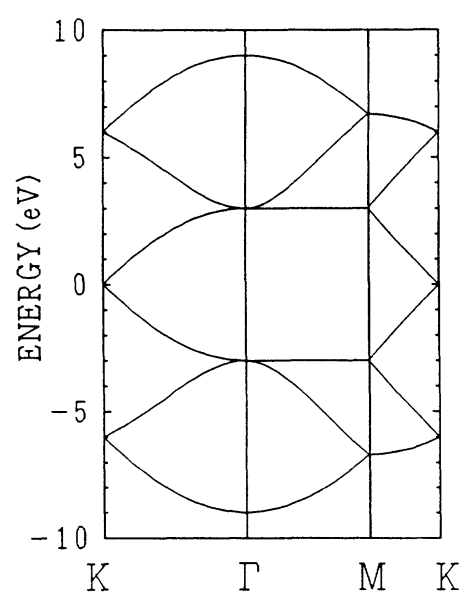

FIG. 7. The folded two-dimensional graphite $\pi$ band in models (a) and (c).

\section{B. Tight-binding models}

\section{Rigid-band model}

In this section, we compare the band structure obtained by the self-consistent calculation in Sec. III A with the rigid band derived from the band structure of graphite. The rigid band of $\mathrm{C}_{4} \mathrm{KH}_{x}$ is obtained by superposing the hydrogen and potassium bands on graphite bands folded into the smaller $\mathrm{BZ}$ of $\mathrm{C}_{4} \mathrm{KH}_{x}$.

First, we present the energy dispersion of graphite. The principal feature of the dispersion of graphite can be understood in terms of a simple tight-binding model of two-dimensional graphite. This model consists of $\pi$ orbitals on two carbon atoms, because the $\pi$ orbitals play a major role in determining the energy dispersion near the Fermi level. In this case, the energy dispersion of 2D graphite is given by an analytical form,

$$
\begin{aligned}
\varepsilon^{ \pm}(\mathbf{k})=\varepsilon_{0} \pm \gamma[ & 1+4 \cos ^{2}\left(\frac{k_{x} a_{0}}{2}\right) \\
& \left.+4 \cos \left(\frac{k_{x} a_{0}}{2}\right) \cos \left(\frac{k_{y} \sqrt{3} a_{0}}{2}\right)\right]^{1 / 2},
\end{aligned}
$$

if we neglect the transfer integrals more distant than nearest neighbors. Here, $a_{0}$ is lattice constant, $\varepsilon_{0}$ is diagonal energy, and $\gamma$ is transfer energy between the nearest-neighbor carbon atoms. The transfer energy $\gamma=3.0 \mathrm{eV}$ suffices to describe the band structure of graphite. As for the diagonal energy, we put $\varepsilon_{0}=0 \mathrm{eV}$. The energy band $\varepsilon^{+}(\mathbf{k})$ corresponds to the upper $\pi$ band ( $\pi^{*}$ band) and $\varepsilon^{-}(\mathbf{k})$ to the lower $\pi$ band.

Next, we discuss the folding of these $\pi$ bands in the hexagonal-lattice model. Figure 6 shows the 2D BZ of $\mathrm{C}_{4} \mathrm{KH}_{x}$ with the $(2 \times 2) R\left(0^{\circ}\right)$ or $(1 \times \sqrt{3}) R\left(0^{\circ}, 30^{\circ}\right)$ lattice models (thick solid line) and the BZ of $2 \mathrm{D}$ graphite (thin solid line). In this figure, the four hatched regions are indicated as $A, B, C$, and $D$. Region $A$ is in the first $\mathrm{BZ}$ of $\mathrm{C}_{4} \mathrm{KH}_{x}$ and regions $B, C$, and $D$ are out of this $\mathrm{BZ}$. Re- 
gions $B, C$, and $D$ are all transferred to region $A$ by proper reciprocal-lattice vectors of $\mathrm{C}_{4} \mathrm{KH}_{x}$. These reciprocal-lattice vectors are shown in Fig. 6.

In Fig. 7, the graphite bands $\varepsilon^{ \pm}(\mathbf{k})$ along the side of regions $A, B, C$, and $D$ are shown together. The rigid band of $\mathrm{C}_{4} \mathrm{KH}_{x}$ is obtained by superposing the hydrogen and potassium bands on this folded graphite band. This band structure is similar to the calculated band structures [Figs. 4(a) and 4(c)].

Consequently, the calculated band structure can be roughly understood in terms of a simple rigid-band model. In the calculated band structure, however, the $\pi^{*}$ bands near the Fermi level split off around the $\Gamma$ point and one of them goes down below the Fermi level. This separation cannot be understood in terms of the rigidband model. We will explain this energy separation of the $\pi^{*}$ bands in Sec. III B 2 using a simple tight-binding model. (In this model we take further account of the charge imbalance between the nonequivalent carbon atoms in a graphite layer.)

Next, we discuss the folding of the $\pi$ bands in the rectangular lattice model. Figure 8 shows the 2D BZ of $\mathrm{C}_{4} \mathrm{KH}_{x}$ with the $(2 \times \sqrt{3}) R\left(0^{\circ}, 30^{\circ}\right)$ rectangular-lattice model (thick solid line) and the BZ of $2 \mathrm{D}$ graphite (thin solid line). The ten hatched regions are indicated as $A$, $B, \ldots, J$. Region $A$ is in the first $\mathrm{BZ}$ of $\mathrm{C}_{4} \mathrm{KH}_{x}$ and regions $B, C, \ldots, J$ are all transferred to region $A$ by proper lattice vectors of $\mathrm{C}_{4} \mathrm{KH}_{x}$. In Fig. 9, the graphite band $\varepsilon^{ \pm}(\mathbf{k})$ along the side of regions $A, B, \ldots, J$ are shown together. This folded band structure is similar to the calculated band structures [Fig. 4(b)].

\section{Separation of $\pi^{*}$ bands near the Fermi level}

In this section, we discuss the energy separation of $\pi^{*}$ bands of $\mathrm{C}_{4} \mathrm{KH}_{x}$ near the Fermi level around the $\Gamma$ point, using a simple tight-binding model. Further, we compare this energy separation with that in the binary GIC $\mathrm{C}_{8} \mathrm{~K}$. In this tight-binding model, we consider only $\pi$ orbitals

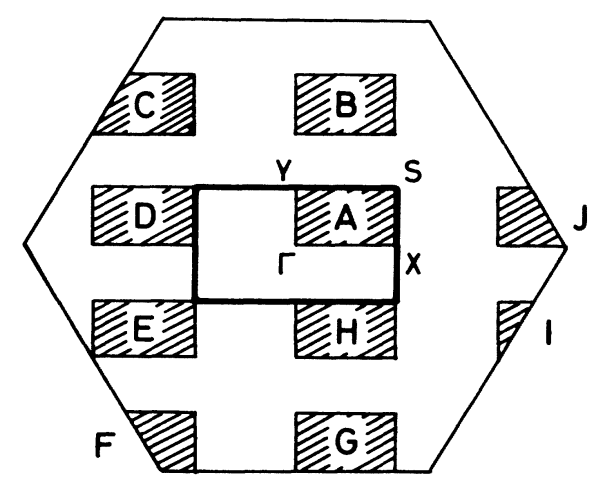

FIG. 8. The 2D BZ of $\mathrm{C}_{4} \mathrm{KH}_{x}$ with the $(2 \times \sqrt{3}) R\left(0^{\circ}, 30^{\circ}\right)$ rectangular-lattice model (thick solid line) and the $\mathrm{BZ}$ of $2 \mathrm{D}$ graphite (thin solid line). The ten hatched regions are indicated as $A, B, \ldots, J$. Region $A$ is in the first $\mathrm{BZ}$ of $\mathrm{C}_{4} \mathrm{KH}_{x}$ and regions $B, C, \ldots, J$ are all transferred to region $A$ by proper lattice vectors of $\mathrm{C}_{4} \mathrm{KH}_{x}$.

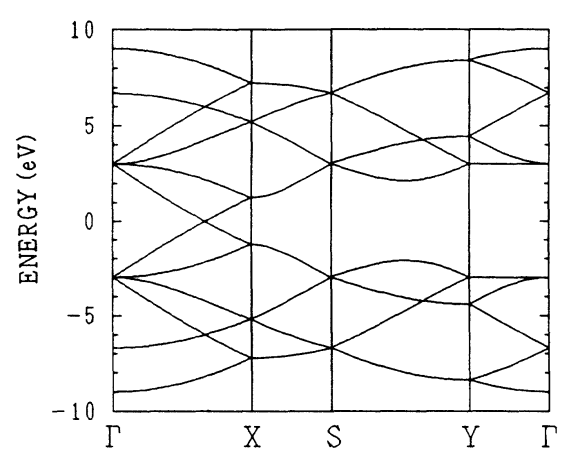

FIG. 9. The folded two-dimensional graphite $\pi$ band in model (b).

on carbon atoms in a graphite layer and neglect the transfer energy more than the nearest neighbor.

Our band-structure calculations (Sec. III A) show that the hydrogen atom acts as an acceptor to graphite while the potassium atom acts as a donor. In our tight-binding model, we assume that the only role played by the potassium atom and the hydrogen atom is to produce the charge transfer between these intercalant and the graphite layer. If we consider only the potassium layers adjacent to the graphite layer, carbon atoms are classified into two environmentally nonequivalent types ( $A$ and $B$ ). In our tight-binding model, we consider only these two types. These are shown in Fig. 10. The $A$-type carbon has two nearest-neighbor potassium atoms, while the $B$ type carbon has one nearest-neighbor potassium atom. Our calculated results (Sec. III A) show that the charge imbalance between the nonequivalent carbon atoms is produced. In order to consider this charge imbalance, we use in our tight-binding model five parameters: $\varepsilon_{A}, \varepsilon_{B}$, $\gamma_{A A}, \gamma_{A B}$, and $\gamma_{B B}$. Here, the parameters $\varepsilon_{A}$ and $\varepsilon_{B}$ are the diagonal energy of the $A$-type and $B$-type carbons, respectively. The parameters $\gamma_{A A}, \gamma_{A B}$, and $\gamma_{B B}$ are

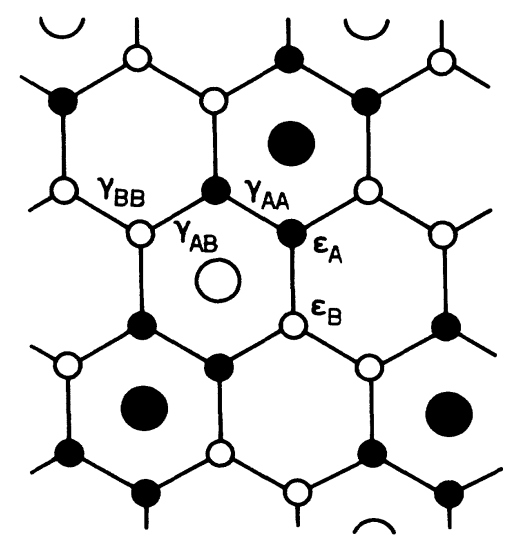

FIG. 10. Two types of carbon. Here, closed and open circles represent the $A$-type and $B$-type carbons, respectively. The $A$ type carbon has two nearest potassium atoms, while the $B$-type carbon has only one. 
transfer energy between the $A$-type carbons, the $A$-type and $B$-type carbons, and the $B$-type carbons, respectively (Fig. 10).

For the sake of simplicity, we assume that

$$
\begin{aligned}
& \varepsilon_{A}=-\frac{1}{2} \varepsilon, \\
& \varepsilon_{B}=+\frac{1}{2} \varepsilon,
\end{aligned}
$$

and

$$
\gamma_{A A}=\gamma_{A B}=\gamma_{B B}=\gamma .
$$

Then, $\varepsilon$ and $\gamma$ are parameters. As for the parameter $\gamma$, we use the transfer energy between the nearest-neighbor carbon in the 2D graphite (see Sec. III B 1). That is, we set $\gamma=3.0 \mathrm{eV}$.

When $\varepsilon=0$, the energy dispersion agrees with the folded $\pi$ band in Fig. 7 (Sec. III B 1). When $\varepsilon \neq 0$, the $\pi^{*}$ bands split off around the $\Gamma$ point. Figure 11 shows the energy dispersion in the case of $\varepsilon=0.5 \mathrm{eV}$. This is similar to the feature in the calculated band structure [Figs. 4(a) and 4(c)]. Therefore, the feature of the separation of $\pi^{*}$ bands around the $\Gamma$ point is well reproduced by using this two-parameter tight-binding model.

In our previous work, ${ }^{17,18}$ we calculated the band structure of the binary GIC $\mathrm{C}_{8} \mathrm{~K}$ and found that one of the $\pi^{*}$ bands goes down below the Fermi level. However, the feature of this separation is different from that in $\mathrm{C}_{4} \mathrm{KH}_{x}$. It cannot be understood in terms of the present two-parameter tight-binding model.

In the next step, we use the three-parameter model. Because we now consider only the $\pi$ orbitals, it is expected that the transfer integral between the same type of $\pi$ orbitals has a larger value than that between the different types of $\pi$ orbitals. Therefore, we assume that

$$
\begin{aligned}
& \gamma_{A A}=\gamma_{B B}=\gamma(=3.0 \mathrm{eV}), \\
& \gamma_{A B}=\gamma-\delta .
\end{aligned}
$$

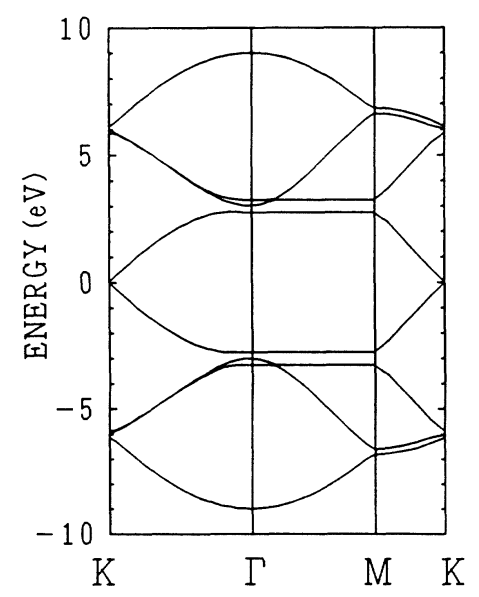

FIG. 11. The energy dispersion obtained by a simple tightbinding model. $(\varepsilon=0.5 \mathrm{eV}, \gamma=3.0 \mathrm{eV}$, and $\delta=0 \mathrm{eV}$.)

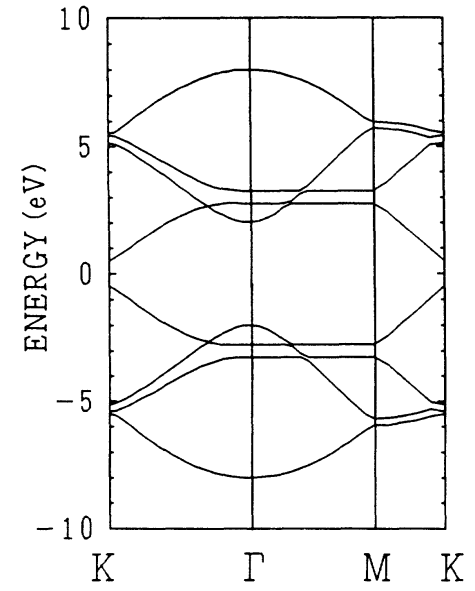

FIG. 12. The energy dispersion obtained by a simple tightbinding model. $(\varepsilon=0.5 \mathrm{eV}, \gamma=3.0 \mathrm{eV}$, and $\delta=0.5 \mathrm{eV}$.)

Figure 12 shows the energy dispersion in the case of $\varepsilon=0.5 \mathrm{eV}$ and $\delta=0.5 \mathrm{eV}$. In this case, the feature of the separation of the $\pi^{*}$ bands in $\mathrm{C}_{8} \mathrm{~K}$ is well reproduced. Therefore, the difference between the feature of the $\pi^{*}$ band separation in $\mathrm{C}_{8} \mathrm{~K}$ and that in $\mathrm{C}_{8} \mathrm{KH}_{x}$ can be understood in terms of the difference of the parameter $\delta$. The amount of the charge imbalance between the nonequivalent carbon in $\mathrm{C}_{8} \mathrm{~K}$ is large (this is about 0.5 in our previous calculation). Thus, the difference $\delta$ between $\gamma_{A A}$ $\left(\gamma_{B B}\right)$ and $\gamma_{A B}$ is important in $\mathrm{C}_{8} \mathrm{~K}$. On the other hand, the difference between $\gamma_{A A}$ and $\gamma_{B B}$ is not so important. When $\gamma_{A A}>\gamma_{B B}$ or $\gamma_{A A}<\gamma_{B B}$, the $\pi^{*}$ band obtained by the tight-binding model has essentially the same feature as in $\gamma_{A A}=\gamma_{B B}$.

In this section, we discussed the energy separation of the $\pi^{*}$ bands of $\mathrm{C}_{4} \mathrm{KH}_{x}$ and $\mathrm{C}_{8} \mathrm{~K}$ near the Fermi level around the $\Gamma$ point, using simple tight-binding models. We found that the charge imbalance between the nonequivalent carbon atoms in a graphite layer is important for understanding this separation, and the difference between the feature of the $\pi^{*}$ band separation in $\mathrm{C}_{8} \mathrm{~K}$ and that in $\mathrm{C}_{8} \mathrm{KH}_{x}$ is due to the amount of the difference between the transfer energy $\gamma_{A A}\left(\gamma_{B B}\right)$ and $\gamma_{A B}$.

\section{SUMMARY}

In conclusion, we have found that the $\mathrm{H} 1 s$ state forms a weakly metallic band in $\mathrm{C}_{4} \mathrm{KH}_{x}$, as in $\mathrm{C}_{8} \mathrm{KH}_{x}$. We estimated the amount of the charge redistribution among the individual atoms and found that the hydrogen atom acts as an acceptor, while the potassium atom acts as a donor. The charge transfer to the hydrogen atom is imperfect. As a result, the $\mathrm{H} 1 s$-like band coexists with the graphite $\pi^{*}$-like bands at the Fermi level. These results indicate that $\mathrm{C}_{4} \mathrm{KH}_{x}$ has a feature of the metallic hydrogen.

The overall band structure of $\mathrm{C}_{4} \mathrm{KH}_{x}$ is roughly understood in terms of the rigid-band model. However, the 
separation of the $\pi^{*}$ bands near the Fermi level cannot be understood in terms of this model. We use a simple tight-binding model and find that the charge imbalance between the nonequivalent carbon atoms in a graphite layer is important for understanding this separation. Further, it is found that the difference between the feature of the $\pi^{*}$ band separation in $\mathrm{C}_{8} \mathrm{~K}$ and that in
$\mathrm{C}_{8} \mathrm{KH}_{x}$ is due to the amount of the difference between the transfer energy $\gamma_{A A}\left(\gamma_{B B}\right)$ and $\gamma_{A B}$.

\section{ACKNOWLEDGMENTS}

We would like to thank Dr. Hisashi Hiramoto for valuable discussions.
${ }^{1}$ T. Enoki, M. Sano, and H. Inokuchi, Phys. Rev. B 32, 2497 (1985).

${ }^{2}$ T. Enoki, N. C. Yeh, S. T. Chen, and M. S. Dresselhaus, Phys. Rev. B 33, 1292 (1986).

${ }^{3}$ G. L. Doll, M. H. Yang, and P. C. Eklund, Phys. Rev. B 35, 9790 (1987).

${ }^{4}$ K. Nomura, T. Saito, K. Kume, and H. Suematsu, Solid State Commun. 63, 1059 (1987).

${ }^{5}$ S. Miyajima, T. Chiba, T. Enoki, H. Inokuchi, and M. Sano, Phys. Rev. B 37, 3246 (1988).

${ }^{6}$ T. Enoki, K. Imada, H. Inokuchi, and M. Sano, Phys. Rev. B 35, 9399 (1987).

${ }^{7}$ T. Saito, K. Nomura, K. Mizoguchi, K. Kume, and $\mathrm{H}$. Suematsu, J. Phys. Soc. Jpn. 58, 269 (1989).

${ }^{8}$ D. Guerard, N. E. Elalem, and C. Takoudjou, Synth. Met. 12, 195 (1985).

${ }^{9}$ W. A. Kamitakahara, G. L. Doll, and P. C. Eklund (unpub- lished).

${ }^{10}$ D. Guerard, C. Takoudjou, and F. Rousseaux, Synth. Met. 7, 43 (1983).

${ }^{11}$ T. Trewern, R. K. Thomas, G. Naylor, and W. White, J. Chem. Soc. Faraday Trans., Ser. 1, 78, 2369 (1983).

${ }^{12}$ S. Mizuno and K. Nakao, Phys. Rev. B 40, 5771 (1989).

${ }^{13}$ S. Mizuno and K. Nakao, J. Phys. Soc. Jpn. 58, 3679 (1989).

${ }^{14}$ P. Hohenberg and W. Kohn, Phys. Rev. 136, B864 (1964).

${ }^{15}$ W. Kohn and L. J. Sham, Phys. Rev. 140, A1133 (1965).

${ }^{16}$ A. Zunger and A. J. Freeman, Phys. Rev. B 15, 4716 (1977).

${ }^{17}$ S. Mizuno, H. Hiramoto, and K. Nakao, Solid State Commun. 63, 705 (1987).

${ }^{18}$ S. Mizuno, H. Hiramoto, and N. Nakao, J. Phys. Soc. Jpn. 56, 4466 (1987).

${ }^{19}$ L. Hedin and B. I. Lindqvist, J. Phys. C 4, 2064 (1971).

${ }^{20}$ S. Miyajima, M. Kabasawa, T. Chiba, T. Enoki, and $\mathrm{H}$. Inokuchi, Phys. Rev. Lett. 64, 319 (1990). 